\title{
Extracellular Matrix Proteins Modulate Antimigratory and Apoptotic Effects of Doxorubicin
}

\author{
Georges Said, ${ }^{1}$ Marie Guilbert, ${ }^{1}$ Hamid Morjani, ${ }^{1}$ Roselyne Garnotel, ${ }^{2}$ \\ Pierre Jeannesson, ${ }^{1}$ and Hassan El Btaouri ${ }^{3}$ \\ ${ }^{1}$ UFR Pharmacie, FRE CNRS/URCA no. 3481, Université de Reims Champagne-Ardenne, 51096 Reims, Cedex, France \\ ${ }^{2}$ UFR Medecine, FRE CNRS/URCA no. 3481, Université de Reims Champagne-Ardenne, 51096 Reims, Cedex, France \\ ${ }^{3}$ UFR Sciences, FRE CNRS/URCA no. 3481, Université de Reims Champagne-Ardenne, 51687 Reims, Cedex 2, France
}

Correspondence should be addressed to Hassan El Btaouri, hassan.elbtaouri@univ-reims.fr

Received 3 February 2012; Accepted 30 April 2012

Academic Editor: Vassilios A. Georgoulias

Copyright () 2012 Georges Said et al. This is an open access article distributed under the Creative Commons Attribution License, which permits unrestricted use, distribution, and reproduction in any medium, provided the original work is properly cited.

\begin{abstract}
Anticancer drug resistance is a multifactorial process that includes acquired and de novo drug resistances. Acquired resistance develops during treatment, while de novo resistance is the primary way for tumor cells to escape chemotherapy. Tumor microenvironment has been recently shown to be one of the important factors contributing to de novo resistance and called environment-mediated drug resistance (EMDR). Two forms of EMDR have been described: soluble factor-mediated drug resistance (SFM-DR) and cell adhesion-mediated drug resistance (CAM-DR). Anthracyclines, among the most potent chemotherapeutic agents, are widely used in clinics against hematopoietic and solid tumors. Their main mechanism of action relies on the inhibition of topoisomerase I and/or II and the induction of apoptosis. Beyond this well-known antitumor activity, it has been recently demonstrated that anthracyclines may display potent anti-invasive effects when used at subtoxic concentrations. In this paper, we will describe two particular modes of EMDR by which microenvironment may influence tumor-cell response to one of these anthracyclines, doxorubicin. The first one considers the influence of type I collagen on the antimigratory effect of doxorubicin (CAM-DR). The second considers the protection of tumor cells by thrombospondin-I against doxorubicin-induced apoptosis (SFM-DR).
\end{abstract}

\section{Tumor Cell Microenvironment and Drug Resistance}

In the last decade, the earlier point of view of tumors as a relatively homogeneous cancer cells has been totally changed into considering tumors as high complex organs. The autonomous properties of cancer cells are no longer sufficient to elucidate the multistep process of tumorogenesis. In fact, a better understanding of this process needs to take into consideration the stromal cells and the extracellular matrix (ECM) proteins that constitute the tumor microenvironment. These elements are known to contribute to the development and the expression of certain cancer hallmarks [1].

It is well documented that anticancer drug resistance represents a major obstacle for the successful treatment of various human malignancies. This process is multi- factorial and can be subdivided into two broad categories: acquired and environment-mediated drug resistance (EMDR). Acquired resistance develops during treatment as a result of sequential genetic changes leading mainly to overexpression of drug transporters and alterations in drug targets [2]. Conversely, EMDR a form of de novo resistance allows cancer cells to tolerate the stress induced by therapies during the first exposure to anticancer drugs. It represents the primary way for tumor cells to escape the cytotoxic effect of anticancer drugs and could be therefore a potential target to overcome resistance to chemotherapy. Two forms of EMDR have been recently described; they are rapidly induced by signaling events resulting from direct cell contact with tumor microenvironment. The first, socalled the soluble factor-mediated drug resistance (SFM-DR) is induced by mediators such as cytokines, chemokines, and growth factors secreted by both tumor and stromal cells. The 
second, the cell adhesion-mediated drug resistance (CAMDR) is mediated by the adhesion of tumor cell integrins to microenvironmental factors such as ECM components (collagen, fibronectin, and laminin) and ligands expressed on stromal cells especially cancer-associated fibroblasts. CAM-DR has been extensively described to confer potential resistance in leukemic and solid tumor cell lines to various chemotherapeutic agents [3].

Anthracyclines, one of the most potent classes of chemotherapeutic agents, are widely used in clinics against hematopoietic and solid tumors. Their main mechanism of action relies on the induction of cytotoxic or apoptotic effects. Indeed, in a wide panel of cancer cell lines, these drugs have been shown to trigger apoptosis via endogenous ceramide increase, mitochondrial and caspases pathways [4]. Beyond this well-known antitumor activity, it has been recently demonstrated in conventional cell culture on plastic substrate that they may display potent anti-invasive effects when used at subtoxic concentrations. Doxorubicin and related compounds such as aclacinomycin and DA-125, a doxorubicin analogue, have been shown to inhibit in vitro invasion of tumor cell lines originating from various solid tumors (prostate, breast, fibrosarcoma, etc.). This antiinvasive effect involves downregulation of matrix metalloproteinases (MMPs) [5], disorganization of cytoskeleton and focal adhesion contacts via inhibition of focal adhesion kinase (FAK) [6]. However, the primary pharmacological target of such an anti-invasive effect is still to be determined.

In this paper, we will describe two particular modes of EMDR by which microenvironment may influence tumor cell response to one of these anthracyclines, doxorubicin. The first considers the influence of type I collagen on the antimigratory effect of doxorubicin in HT1080 human fibrosarcoma cells (CAM-DR). The second considers the protection of human thyroid carcinoma FTC-133 cells by thrombospondin-I against doxorubicin-induced apoptosis (SFM-DR).

\section{Influence of Type I Collagen on the Antimigratory Effect of Doxorubicin}

2.1. Collagen-Based Cell Migration Models. In humans, type I collagen is the main component of ECM in connective tissues, through which tumor cells usually move to form metastasis and use as a preintravasation microenvironment [7]. Type I collagen is characterized by a triple-helical structure, two $\alpha_{1}$ and one $\alpha_{2}$ chains, which are stabilized by the formation of cross-links between and within the triplehelices, allowing it to form a fibrillar network. It mediates the biomechanical stability of connective tissue and provides a 3D scaffold to which other ECM proteins such as fibronectin and glycosaminoglycans are connected [8].

Early in vitro studies of cell adhesion and migration have been performed using two-dimensional (2D) rigid, planar substrates, coated with different types of ECM proteins such as type I collagen or fibronectin. Recently, these conventional cell migration models have given place to reconstituted $3 \mathrm{D}$ collagen matrices. These scaffolds offer both more realistic view due to the coupling of chemical and mechanical signals that take place in the real tissues and better simulation of cell response to anticancer drugs [9]. We here employed processed adult rat tail collagen at a concentration of $1.5 \mathrm{mg} / \mathrm{mL}$ known to be close to that found in vivo [10]. The highly fibrillar organization of this matrix therefore approximates the 3D fibrous nature of mesenchymal stroma [11]. In addition, due to its preparation without pepsinization, this native fibrillar collagen presents intact telopeptides in contrast with most of the experimentally generated collagen matrices currently using pepsin-cleaved type I collagen [12]. Telopeptides which correspond to the flanking regions of the molecules permit to form intra- and intermolecular cross-links that promote the staggering and the resilience of fibrillar collagen [13]. Such 3D models are well suited to directly analyze the migratory behavior and the morphology of individual moving cells by using time-lapse videomicroscopy. The in situ cell cytoskeleton organization after actin staining and the proliferative capacity following collagenase treatment can be also estimated. In addition, culturing tumor cells in $3 \mathrm{D}$ is a well-adapted approach for evaluating the cytotoxic and anti-invasive effects of anticancer drugs with the condition that their diffusion capacities have been previously tested through matrices. Indeed, drug penetration into matrices strongly depends on their molecular weight as demonstrated for the low diffusion rate of antibodies [14] compared to unaffected diffusion rate of doxorubicin representative of low molecular weight drugs [11].

\subsection{Type I Collagen Protects Tumor Cells against the Antimi-} gratory Effect of Doxorubicin. The influence of type I collagen on the antimigratory effect of doxorubicin was investigated by using the highly invasive fibrosarcoma cell line HT1080. At drug concentrations exhibiting no or limited effect on tumor cell proliferation, the ability of doxorubicin to decrease tumor cell motility was studied in $2 \mathrm{D}$ and $3 \mathrm{D}$ collagen-based systems. For this purpose, tumor cells were cultured either on $2 \mathrm{D}$ coatings [15] or within $3 \mathrm{D}$ matrices of type I collagen [16] and in parallel conventional 2D cultures were performed by seeding cells on plastic substrata. Exploring the role of doxorubicin at subtoxic concentrations permitted to exclude that the antimigratory effect of the drug could result from a nonspecific consequence of its cytotoxic effect. This is also of interest for therapeutic application since the use of doxorubicin is often limited by severe cardiocytotoxicity and other side effects [17].

On conventional plastic surfaces, the subtoxic concentrations of doxorubicin induced a marked inhibition of HT1080 cell migration as demonstrated by time-lapse videomicroscopy. Individual cell velocity is decreased but the frequency and mean length of breaks were not affected. In addition, cell trajectories were profoundly modified since cells exhibited shorter or circular paths around their starting point in accordance with cell speed inhibition. These results indicate that the antimigratory effect preferentially takes place during the locomotory and not the stationary phase of the cells. By contrast, this deleterious effect on cell velocity 

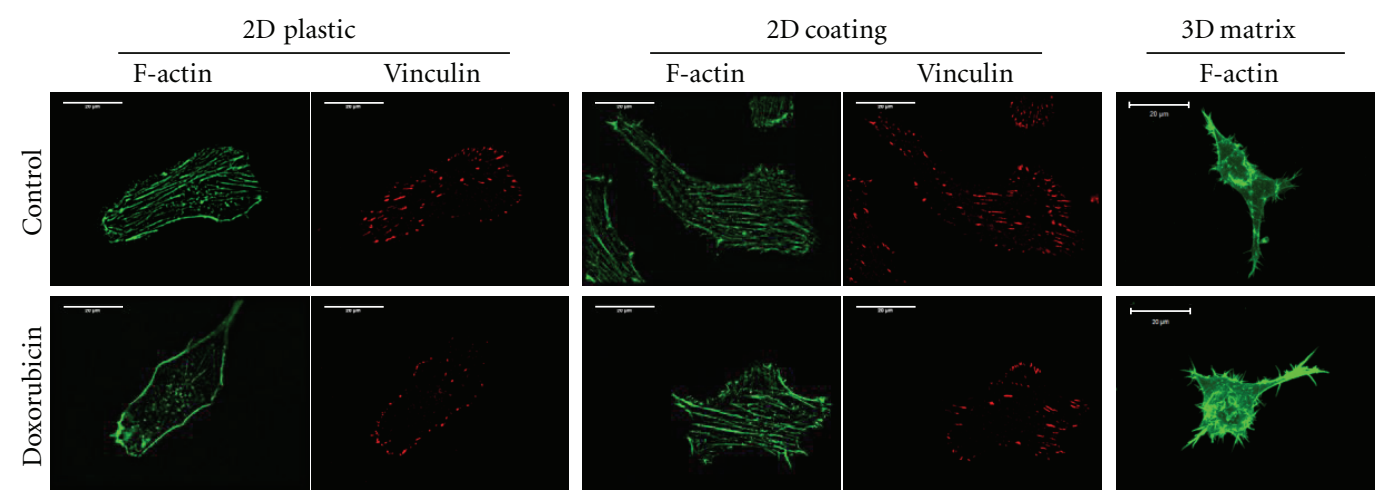

FIGURE 1: Type I collagen protects HT1080 cells against the doxorubicin-induced disorganization of cytoskeleton. After $24 \mathrm{~h}$ of exposure to subtoxic concentrations of doxorubicin $(5$ and $10 \mathrm{nM})$, cells were stained for F-actin or vinculin. $($ Bar $=20 \mu \mathrm{m})$.

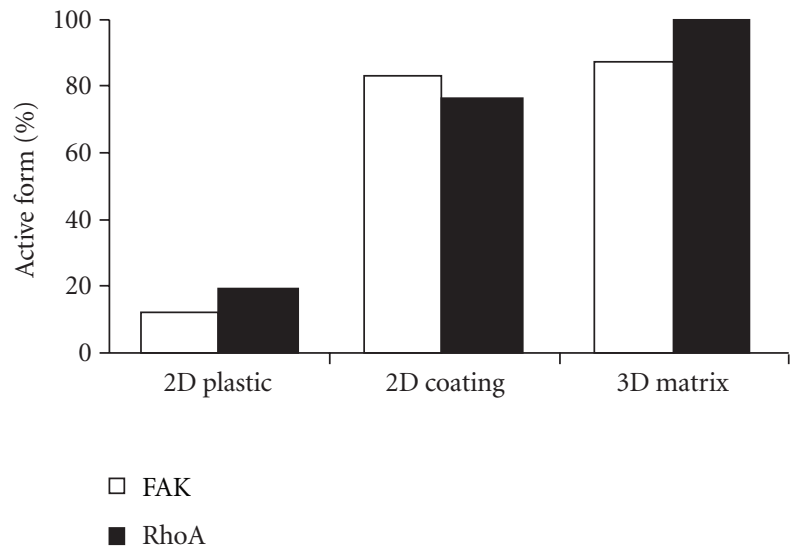

FIGURE 2: Type I collagen protects HT1080 cells from doxorubicininduced dephosphorylation of FAK and RhoA. After $24 \mathrm{~h}$ of exposure to doxorubicin ( 5 and $10 \mathrm{nM}$ ), cells cultured on plastic or 2D coated type I collagen were directly lyzed, except for those cultured inside 3D matrices that were beforehand digested by collagenase P. The expression and the activation state of FAK and RhoA were quantified by western blot. $Y$-axis corresponds to the percentage ratio of active form of FAK or RhoA in doxorubicintreated cells with respect to untreated cells.

and trajectories was totally abolished when doxorubicintreated cancer cells were cultured, respectively, on type I collagen-coated surfaces or inside 3D type I collagen matrices (Table 1). Such results are completely in agreement with inhibitory effects of ECM components on the cytotoxic mechanism of various anticancer drugs [18].

\subsection{Type I Collagen Abolishes Doxorubicin Effects on the} Migratory Molecular Regulators. Cell migration is a complex multistep process involved in the movement of cells from one location to another contributing therefore to cancer metastasis. The formation of new adhesions via the assembly of focal adhesion complexes and the remodeling of the cytoskeleton via actin stress fiber recruitment appear as key mechanisms that regulate cell motility. On plastic surfaces, doxorubicin induced a dramatic disturbance of adaptor proteins of the focal adhesion complexes such as vinculin and FAK (Figures 1 and 2). This indicates that doxorubicin acts through altering signaling complexes normally involved in the signal transmission from the ECM to the cell cytoskeleton, which could constitute the primary targets of its antimigratory effect. In presence of the drug, vinculin, an actin binding protein recruited to the $\beta$ integrin cytoplasmic tail, is delocalized from the end of each stress fibers to the rim of the cells. In addition, the drug strongly inhibited the phosphorylation state of FAK on $\mathrm{Tyr}^{397}$ without modifying its expression, altering therefore FAK signaling. Concerning the cytoskeleton, doxorubicin induces complete disorganization of actin with dramatic loss of stress fibers (Figure 1). This is accompanied by a marked inhibition of the phosphorylation state of the GTPase RhoA, a key protein involved in the regulation of actin stress fiber formation. By contrast, with collagen in 2D and 3D conditions, these effects on cytoskeleton organization and on the activation state of FAK and RhoA were completely abolished (Figures 1 and 2). Taken together, these data demonstrate that type I collagen is able to protect tumor cells against the anti-invasive effect of anthracyclines.

\section{Modulation of Chemotherapy-Induced Apoptosis by Extracellular Matrix Components}

Several studies have progressively shown the role of the ECM in the modulation of the cell response to chemotherapy (survival/resistance) $[3,20,21]$. It is admitted now that the modulation of cell response to chemotherapy by ECM contributes to a new form of de novo resistance. The group of W. S. Dalton was the first to show that ECM is able to confer resistance to chemotherapy [3, 22, 23]. They demonstrated the role of fibronectin in tumor cell protection against chemotherapy via $\beta 1$ integrin $[24,25]$. This protection occurs through the activation of PI 3-kinase signaling pathway [26]. This property has been also reported for vitronectin via $\alpha v \beta 3$ and $\alpha \mathrm{v} \beta 5$ integrin [27]. By contrast, other components of the ECM are able to sensitize tumor cells to the cytotoxic effect of anticancer drugs. TGF $\beta 1$ is 


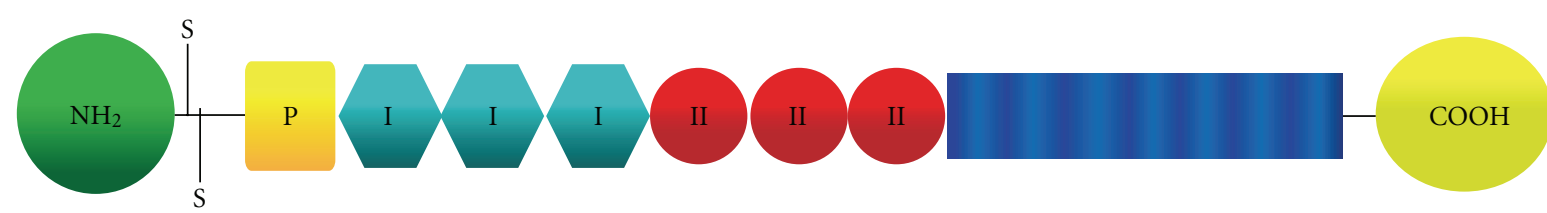

\begin{tabular}{|c|c|c|c|c|c|}
\hline $\begin{array}{c}\text { Amino- } \\
\text { terminal } \\
\text { domain }\end{array}$ & $\begin{array}{c}\text { Procollagen } \\
\text { homology } \\
\text { domain }\end{array}$ & $\begin{array}{c}\text { Type I repeat } \\
\text { domains }\end{array}$ & $\begin{array}{c}\text { Type II repeat } \\
\text { domains }\end{array}$ & $\begin{array}{c}\text { Carboxy- } \\
\text { terminal } \\
\text { domain }\end{array}$ \\
\hline
\end{tabular}

\begin{tabular}{|c|c|c|c|}
\hline TSP1 Domains & Sequences & Receptors or ligands & Functions \\
\hline & \multirow{5}{*}{ RKGSGRR/KKTR } & \multirow[t]{2}{*}{$\alpha 3 \beta 1$ integrin } & Cell adhesion \\
\hline & & & Hemostasis \\
\hline & & HSPG-LRP-gp330 & Endocytosis \\
\hline & & Syndecan & \\
\hline & & Sulfated glycolipid & \\
\hline
\end{tabular}

$\begin{array}{cccc}\begin{array}{l}\text { Amino-terminal } \\ \text { domain }\end{array} & \text { MKKTRG } & \text { Decorin } & \text { Inhibition of cell adhesion } \\ \text { BBXB } & \text { Heparin } & \text { Cell adhesion } \\ \text { LDVP } & \alpha 4 \beta 1 \text { integrin } & \text { Promote cell adhesion } \\ & \alpha 5 \beta 1 \text { integrin } & \text { Chemotaxis } \\ & & \text { MMP synthesis stimulation } \\ 25 \mathrm{kDa} \text { peptide } & ? & \text { Angiogenesis stimulation }\end{array}$

\begin{tabular}{|c|c|c|c|}
\hline & \multirow{2}{*}{ Multimerization } \\
\hline $\begin{array}{l}\text { Oligomerization } \\
\text { domain }\end{array}$ & & & \\
\hline Procollagen homologs & NGVQYRN & & Angiogenesis inhibition \\
\hline domain & & & Chemotaxis inhibition \\
\hline \multirow{7}{*}{ Type I repeat domains } & RFK & LTGF $\beta$ & Latent TGF $\beta$ activation \\
\hline & WSHWSPW & Protein & Latent TGF $\beta$ activation \\
\hline & & glycosaminoglycan & \\
\hline & GGWSHW & Fibronectin & Cell adhesion \\
\hline & CSVTCG & CD36 & Anti-angiogenesis \\
\hline & & HIV gp 120 & Cell adhesion \\
\hline & & HSPG & \\
\hline \multicolumn{4}{|l|}{$\begin{array}{l}\text { Type II repeat } \\
\text { domains }\end{array}$} \\
\hline \multirow{7}{*}{$\begin{array}{l}\text { Type III repeat } \\
\text { domains }\end{array}$} & RGD & $\alpha \mathrm{v} \beta 3$ integrin & Cell adhesion \\
\hline & & $\alpha \operatorname{llb} \beta 3$ integrin & \\
\hline & & & Calcium binding \\
\hline & NCPFHYNP & Cathepsin G, elastase & \\
\hline & NCQYVYNV & Elastase & \\
\hline & RFYVVM & $\mathrm{CD} 47$ & Chemotaxis \\
\hline & IRVVM & & Cell proliferation \\
\hline
\end{tabular}

FIgURE 3: Schematic representation of the multimodular structure and functions of a single subunit of the TSP-1. TSP-1 presents several distinct domains with specific biological properties. In the table are indicated the known peptide sequences, the corresponding receptors or ligands, and the associated biological activities [19].

able to sensitize ovarian carcinoma cells to paclitaxel [28]. In this case, it has been clearly demonstrated that low TGF $\beta 1$ expression could be a poor prognosis in patients [29].

Thrombospondin-1 (TSP-1) is known for playing role in the induction of apoptosis in endothelial cells via the CD36 receptor [30]. Besides this antiangiogenic effect, this protein is also able to modulate the response of tumor cells to chemotherapy via the CD47 receptor. As mentioned for TFG $\beta 1$, TSP- 1 is also able to sensitize prostate carcinoma cells to the cytotoxic effect of taxol $[31,32]$. Clinical data confirmed the in vitro model and demonstrated that tumoral expression of TSP-1 predicts overall survival of patients with lung adenocarcinoma treated with first-line docetaxelgemcitabine regimen [33]. Other authors have demonstrated 
that cisplatin was able to reverse resistance to taxol in nasopharyngeal carcinoma by upregulating TSP-1 expression [34]. Akiyamas group has reported that 5-fluorouracil (5FU) induced TSP-1 in human colon carcinoma cells. A transcription factor, Egr-1, was also induced by 5-FU and bound to the promoter of TSP-1, enhancing its transcription and the subsequent production of TSP-1 protein. Moreover, this group presented the evidence that p38 mitogen-activated protein kinase (MAPK) plays an important role in 5-FUinduced Egr-1 transactivation [35]. Because estrogens cause progression of many breast cancers, Wu laboratory have examined whether TSP-1 is regulated by estrogen. Estradiol (E2) induced TSP-1 expression in human breast cancer cells in vitro. This induction was blocked by the estrogen antagonists, indicating that estrogen receptors are necessary for this effect. Furthermore, E2 caused the production of TSP-1 protein from tumor cells in an ER- $\alpha$-dependent manner [36]. The same authors have shown that synthetic progestins also induce TSP-1 mRNA and protein in human breast cancer cells. Antiprogestin RU-486 was able to inhibit the induction of TSP-1 by progestins, suggesting also that this effect is mediated by the progesterone receptor [37].

Our group has reported recently that doxorubicin is able to induce apoptosis in thyroid carcinoma cells via ceramide de novo synthesis [38]. Moreover, this apoptosis is accompanied by a downregulation of TSP-1 expression at mRNA and protein levels [39]. The addition of TSP1 protects the cells against doxorubicin-induced apoptosis [39]. The antiapoptotic role of TSP-1 involves its C-terminal part that interacts with the membrane receptor CD47. More recently, we have shown that both doxorubicin was able to activate JNK/ATF-2 pathway to downregulate TSP-1 expression and to modulate apoptosis [40]. The role of TSP-1 in the protection of thyroid carcinoma cells will be discussed in more detail in this second part of the paper.

3.1. Structural Organization and Role of TSP-1. TSP-1 is an ECM glycoprotein first discovered in activated platelets [41]. TSP-1 has been the first identified member of the thrombospondin family in 1971 [42] since this protein was released in response to thrombin in activated platelets and participated to the formation of the fibrin clot [43]. Different data are now available about the role of TSP-1 in cancer and support the hypothesis of important functions for TSP-1 in tumor growth and metastasis. However, conflicting results led to consider TSP-1 either as a tumor suppressor or as a tumor promoter [44].

The cascade of signal transduction following interaction between the cell and ECM components constitutes an interesting way of investigation since ECM proteins are recognized as important regulators of cell growth and function. TSP-1 presents multiple structural domains and putative ligand binding sites including integrins, CD36 [45], CD47, low-density lipoprotein (LDL) receptor-relatedprotein and proteoglycans [46]. This diversity implies that TSP-1 can interact at the same time with one or more receptors in a cell type. Otherwise, the cell response to TSP1 can differ according to the respective levels of receptors expression and leads to opposite responses depending on the physiopathological situation.

TSP-1 is expressed on the cell surface during physiological events. A variety of normal cells, including endothelial cells, fibroblasts, adipocytes, smooth muscle cells, monocytes, macrophages, and transformed cells such as malignant glioma cells to eliminate secrete TSP-1 [47, 48].

TSP-1 contains a N-terminal globular domain that binds heparin, type I, type II, and type III repeats, and a terminal globular domain. The structure of TSP-1 is schematically shown in Figure 1. The $\mathrm{NH}_{2}$-terminal of TSP-1 interacts with low-density lipoprotein receptor-related protein (LRP1) [49], heparin sulfate proteoglycans and a number of integrins that have an important function in angiogenesis, chemotaxis adhesion, and cell motility [50]. All five members of the TSP family have the repeat domains type II and III, but only TSP-1 and TSP-2 contain the type I repeats [51]. Type I repeats, also called thrombospondin structural homology repeats (TSRs), inhibit angiogenesis by activating CD36 and inducing apoptosis in endothelial cells [52] (Figure 3).

The COOH-terminal domain of TSP-1 binds to CD47, also known as integrin-associated protein [53]. This domain also interacts with integrins such as $\beta 1$ and $\beta 6$ integrins and actively binds to proteoglycans allowing cell adhesion and spreading [50]. These and other interactions significantly affect angiogenesis, cell proliferation, and immune responses (Figure 3).

3.2. TSP-1 and Chemotherapy. Previous studies have developed the concept that the levels of TSP-1 could be directly correlated with the resistance and aggressiveness of the thyroid cancer. Recent data showed that FTC-238 thyroid cells, exhibiting a higher endogenous TSP-1 level than FTC133, were the less sensitive to doxorubicin treatment [54]. In fact, TSP-1 was previously linked to disease recurrence and decreased patient survival $[55,56]$. Some reports investigated TSP-1 expression level in clinical thyroid cancer cases. One study showed no significant difference in mean TSP-1 mRNA expression in in vivo thyroid cancers in comparison to normal specimens [57]. However, another study demonstrated that TSP-1 expression was reduced in correlation with the increasing aggressiveness of different thyroid lesions [58]. Another report evaluating 75 papillary thyroid cancers, demonstrated that TSP-1 expression was inversely correlated with invasiveness [59]. Indeed, patients with TSP-1-negative tumors appeared to present a poor prognosis in colon cancer [60], and overexpression of TSP-1 in mice lacking endogenous TSP-1 was reported to suppress tumor growth [61].

The effects of TSP-1 have been studied in many preclinical tumor models, and mimetic peptides are being tested in cancer clinical trials. Indeed, the interaction of TSP-1 with the nitric oxide pathway seems to be involved in the antiangiogenic mechanisms mediated by TSP-1 TSR-derived peptide in cancers $[62,63]$, and it might also explain the anti-inflammatory effects of this peptide in the colitis model [64]. Studies of prostate cancers indicate that the combined decrease of NF- $\kappa \mathrm{B}$ and increase of TSP-1, modulated by the 


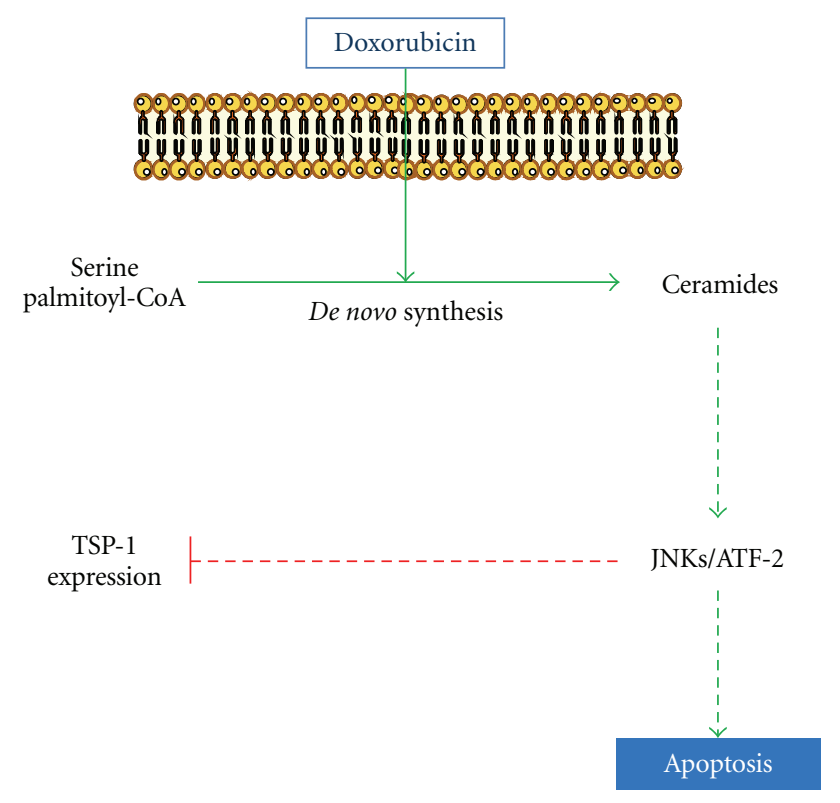

FIGURE 4: Schematic representation of signaling pathway mediated by doxorubicin in inhibition of TSP-1 expression and induction apoptosis.

expression of the androgen receptor, exert antitumor effects [65]. The TSP-1-derived peptide angiocidin has antitumor effects and induces the differentiation of monocytes to macrophages by activating the NF- $\kappa$ B pathway [66].

\subsection{Doxorubicin Induces Apoptosis and Downregulates TSP-} 1 Expression in Human Thyroid Carcinoma FTC-133 Cells. We have reported that doxorubicin, an inhibitor of topoisomerase II, led to elevated cytotoxic events associated with ceramide generation and correlated with TSP-1 down regulation, mainly occurring at the transcriptional level and an induced apoptosis in human thyroid carcinoma FTC-133 cells $[38,39]$.

Ceramide generation can occur through hydrolysis of sphingomyelin, that is, catalyzed by acid or neutral sphingomyelinase or through de novo synthesis starting with serine and palmitate condensation [67-70].

In our study, we demonstrated that neither acid nor neutral sphingomyelinase-dependent activities varied upon doxorubicin treatment in FTC-133 cells. Doxorubicin enhanced ceramide production mainly via the de novo synthesis pathway. This pathway usually results in a prolonged ceramide elevation and was responsible for the drug-induced malignant cell apoptosis through a caspase-3-dependent pathway and a decrease of TSP-1 amount [38] (Figure 4).

C-Jun N-terminal kinases (JNKs) are multifunctional signaling networks that influence cell proliferation, differentiation, apoptosis, and responses to stress playing a pivotal role in the signal transduction from different stimuli [71]. We confirmed that the JNK pathway plays a central role in doxorubicin-induced downregulation of TSP-1 and apoptosis in FTC-133 cells. Moreover, doxorubicin induced JNK phosphorylation through de novo ceramide synthesis and chemical inhibition of either ceramide production or JNK activation prevent doxorubicin effects on TSP1 expression and cell apoptosis. Other reports have also clearly established the role of JNK in X-ray, doxorubicin and TRAIL-induced apoptosis in U937 cells, rat hepatoma cell line, mouse lymphocytic leukemia cells and squamous cell carcinoma, respectively [72-75]. Moreover, JNK inhibition abrogated estradiol-induced suppression of TSP-1 synthesis in endothelial cells [76].

JNK phosphorylates the transcription factors c-Jun, ATF2, Elk-1, p53, and c-Myc, as well as antiapoptotic Bcl2 family members [77]. It has been proposed that JNK-induced ATF-2 phosphorylation might control apoptosis in response to stresses such as UV light, osmotic stress, hypoxia, and inflammatory cytokines $[78,79]$.

Our data demonstrated that ATF-2 was phosphorylated in response to these agents. This phosphorylation was inhibited by SP600125 indicating that JNK controlled ATF-2 activation. Moreover, ATF-2 silencing abolished doxorubicin effects. Other reports have also demonstrated that JNK inhibition led to a decrease of active caspase- 3 and ATF2 phosphorylation which correlated with a decrease in the number of apoptotic cells [80]. In summary, our data put in evidence that JNK/ATF-2 is activated by doxorubicin via de novo ceramide leading to TSP-1 downregulation and consequently apoptosis in FTC-133 cell (Figure 4).

3.4. Anti-apoptotic Effect of TSP-1 in FTC-133 Cells. The correlation between TSP-1 downregulation and apoptosis was confirmed by other investigation underlined that overexpression of the PTEN tumor-suppressor gene leading to induction of thyroid carcinoma apoptosis was also correlated with a significant downregulation of TSP-1 expression at both RNA and protein levels [81]. These results led us to investigate a possible antiapoptotic role for TSP-1 in FTC133 cells. Our results shed new light on the antiapoptotic properties of TSP-1 protein in thyroid cancer. We provided evidences that doxorubicin-induced apoptosis was significantly decreased in the presence of CD47/IAP-binding peptide $4 \mathrm{~N} 1$, a peptide derived from the $\mathrm{COOH}$-terminal domain of TSP-1 [39]. These results were confirmed by antiCD47 antibody that blocked $4 \mathrm{~N} 1$ protective effect [39]. These findings suggest that induction of apoptosis by doxorubicin in FTC-133 cells is dependent on the downregulation of TSP1 expression shedding light on a potential role for TSP-1 in cell response to chemotherapy (Figure 5).

Recent studies described that some cancers may develop the ability to counterbalance their own secretion of proor antitumoral factors [82-84] which could explain the apparent conflicting results. Some tumor cells were shown to bypass the expected inhibitory effect of TSP-1. For example, human breast cancer cells could override the antiangiogenic effects of TSP-1 in vivo by increasing vascular endothelial growth factor (VEGF) expression [85]. In FTC-133 cells, a continuous inhibition of TSP-1 under drug treatment was observed and addition of TSP- 1 or its derived peptide $4 \mathrm{~N} 1$ in doxorubicin-treated cells exhibited an unexpected antiapoptotic effect. Whether other growth factors are implicated 


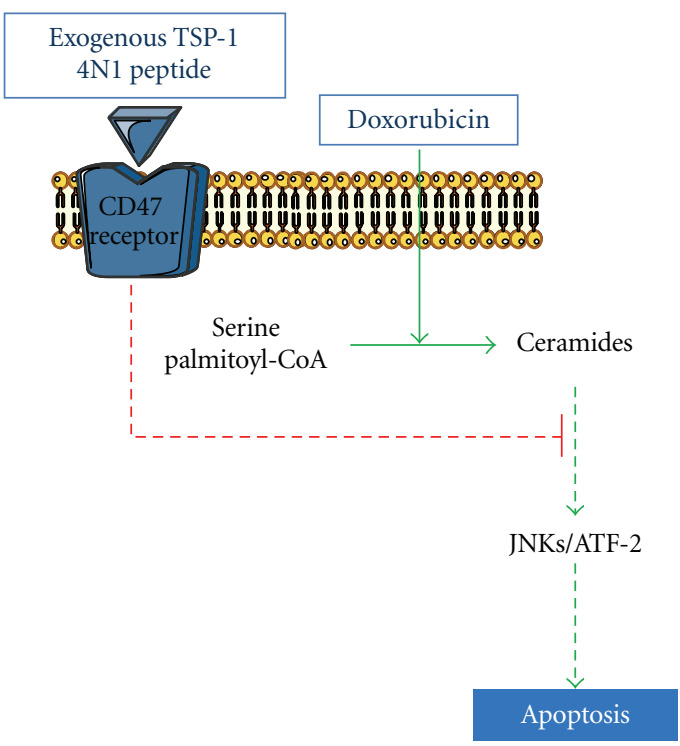

Figure 5: Schematic representation of protective effect of TSP1 C-terminal-derived peptide $4 \mathrm{~N} 1$ against doxorubicin-induced apoptosis in FTC-133 thyroid cells.

TABLE 1: Type I collagen protects HT1080 cells against the antimigratory effect of doxorubicin. The seeded cells on plastic, on $2 \mathrm{D}$ coating or inside $3 \mathrm{D}$ matrices, were exposed to subtoxic concentrations of doxorubicin ( 5 and $10 \mathrm{nM}$ ) for $24 \mathrm{~h}$. Cell motility was examined for the last $12 \mathrm{~h}$ using time-lapse videomicroscopy as described before $[15,16]$.

\begin{tabular}{lccc}
\hline & \multicolumn{3}{c}{ Migration speed $(\mu \mathrm{m} / \mathrm{h})$} \\
& 2D plastic & 2D coating & 3D matrix \\
\hline Control & $15.6 \pm 1.4$ & $19.0 \pm 0.7$ & $24.8 \pm 0.9$ \\
Doxorubicin & $8.3 \pm 0.8$ & $23.0 \pm 1.1$ & $25.7 \pm 1.5$ \\
\hline
\end{tabular}

remains to be demonstrated. Altogether, according to the cellular environment specificity, TSP-1 could be associated with a worse prognosis, and therefore, considered as an interesting marker for patient survival.

Finally, these data shed light on one component of the drug-resistance phenotype in thyroid tumoral diseases. They support the idea that TSP- 1 could be helpful for predicting recurrence and survival outcome in patients affected by such pathologies. Understanding how biological factors such as TSP-1 are capable of modulating tumor-cell response to chemotherapy will be of great interest to enhance therapeutic response and to identify efficient clinical chemotherapeutic protocols.

\section{Concluding Remarks}

ECM proteins were demonstrated to protect tumor cells against the antimigratory and apoptotic effects of antitumor drugs. These data support the crucial role of the tumor microenvironment in the failure of clinical response to chemotherapeutic agents and the emergence of EMDR. In addition, they suggest that tumor cell/ECM interactions should be taken into account in the development of new agents specifically targeting tumor cell proliferation and motility in order to prevent metastasis. More generally, this paper highlights that traditional cell culture models are insufficiently representative of solid tumors, recently considered as complex organs [1]. It suggests that the remodeling of tumor microenvironment could represent an innovative approach [86] to improve therapeutic efficacy of conventional anticancer drugs previously thought to be ineffective.

\section{References}

[1] D. Hanahan and R. A. Weinberg, "Hallmarks of cancer: the next generation," Cell, vol. 144, no. 5, pp. 646-674, 2011.

[2] M. M. Gottesman, "Mechanisms of cancer drug resistance," Annual Review of Medicine, vol. 53, pp. 615-627, 2002.

[3] M. B. Meads, R. A. Gatenby, and W. S. Dalton, "Environmentmediated drug resistance: a major contributor to minimal residual disease," Nature Reviews Cancer, vol. 9, no. 9, pp. 665674, 2009.

[4] G. Laurent and J. P. Jaffrézou, "Signaling pathways activated by daunorubicin," Blood, vol. 98, no. 4, pp. 913-924, 2001.

[5] H. J. Park, H. J. Chung, H. Y. Min et al., "Inhibitory effect of DA-125, a new anthracyclin analog antitumor agent, on the invasion of human fibrosarcoma cells by down-regulating the matrix metalloproteinases," Biochemical Pharmacology, vol. 71, no. 1-2, pp. 21-31, 2005.

[6] S. Addadi-Rebbah, S. Poitevin, N. Fourre, M. Polette, R. Garnotel, and P. Jeannesson, "Assessment of the antiinvasive potential of the anthracycline aclacinomycin (Aclarubicin) in a human fibrosarcoma cell line," International Journal of Oncology, vol. 24, no. 6, pp. 1607-1615, 2004.

[7] I. Serebriiskii, R. Castelló-Cros, A. Lamb, E. A. Golemis, and E. Cukierman, "Fibroblast-derived 3D matrix differentially regulates the growth and drug-responsiveness of human cancer cells," Matrix Biology, vol. 27, no. 6, pp. 573-585, 2008.

[8] K. Wolf, S. Alexander, V. Schacht et al., "Collagen-based cell migration models in vitro and in vivo," Seminars in Cell and Developmental Biology, vol. 20, no. 8, pp. 931-941, 2009.

[9] L. C. Kimlin, G. Casagrande, and V. M. Virador, "In vitro three-dimensional (3D) models in cancer research: an update," Molecular Carcinogenesis. In press.

[10] S. Ramanujan, A. Pluen, T. D. McKee, E. B. Brown, Y. Boucher, and R. K. Jain, "Diffusion and convection in collagen gels: implications for transport in the tumor interstitium," Biophysical Journal, vol. 83, no. 3, pp. 1650-1660, 2002.

[11] N. Fourré, J. M. Millot, R. Garnotel, and P. Jeannesson, "In situ analysis of doxorubicin uptake and cytotoxicity in a $3 \mathrm{D}$ culture model of human HT-1080 fibrosarcoma cells," Anticancer Research, vol. 26, no. 6, pp. 4623-4626, 2006.

[12] F. Sabeh, R. Shimizu-Hirota, and S. J. Weiss, "Proteasedependent versus-independent cancer cell invasion programs: three-dimensional amoeboid movement revisited," Journal of Cell Biology, vol. 185, no. 1, pp. 11-19, 2009.

[13] K. Sato, T. Ebihara, E. Adachi, S. Kawashima, S. Hattori, and S. Irie, "Possible involvement of aminotelopeptide in selfassembly and thermal stability of collagen I as revealed by its removal with proteases," Journal of Biological Chemistry, vol. 275, no. 33, pp. 25870-25875, 2000.

[14] C. D. L. Davies, D. A. Berk, A. Pluen, and R. K. Jain, "Comparison of IgG diffusion and extracellular matrix composition 
in rhabdomyosarcomas grown in mice versus in vitro as spheroids reveals the role of host stromal cells," British Journal of Cancer, vol. 86, no. 10, pp. 1639-1644, 2002.

[15] N. Fourre, E. Millerot-Serrurot, R. Garnotel et al., "Extracellular matrix proteins protect human HT1080 cells against the antimigratory effect of doxorubicin," Cancer Science, vol. 99, no. 8, pp. 1699-1705, 2008.

[16] E. Millerot-Serrurot, M. Guilbert, N. Fourré et al., “3D collagen type I matrix inhibits the antimigratory effect of doxorubicin," Cancer Cell International, vol. 10, article 26, 2010.

[17] M. S. Ewer, D. D. von Hoff, and R. S. Benjamin, "A Historical perspective of anthracycline cardiotoxicity," Heart Failure Clinics, vol. 7, no. 3, pp. 363-372, 2011.

[18] P. J. Morin, "Drug resistance and the microenvironment: nature and nurture," Drug Resistance Updates, vol. 6, no. 4, pp. 169-172, 2003.

[19] B. Sid, H. Sartelet, G. Bellon et al., "Thrombospondin 1: a multifunctional protein implicated in the regulation of tumor growth," Critical Reviews in Oncology/Hematology, vol. 49, no. 3, pp. 245-258, 2004.

[20] S. Hehlgans, M. Haase, and N. Cordes, "Signalling via integrins: implications for cell survival and anticancer strategies," Biochimica et Biophysica Acta, vol. 1775, no. 1, pp. 163-180, 2007.

[21] M. A. Westhoff and S. Fulda, "Adhesion-mediated apoptosis resistance in cancer," Drug Resistance Updates, vol. 12, no. 4-5, pp. 127-136, 2009.

[22] L. A. Hazlehurst, T. H. Landowski, and W. S. Dalton, "Role of the tumor microenvironment in mediating de novo resistance to drugs and physiological mediators of cell death," Oncogene, vol. 22, no. 47, pp. 7396-7402, 2003.

[23] M. B. Meads, L. A. Hazlehurst, and W. S. Dalton, "The bone marrow microenvironment as a tumor sanctuary and contributor to drug resistance," Clinical Cancer Research, vol. 14, no. 9, pp. 2519-2526, 2008.

[24] L. A. Hazlehurst, R. F. Argilagos, M. Emmons et al., "Cell adhesion to fibronectin (CAM-DR) influences acquired mitoxantrone resistance in U937 cells," Cancer Research, vol. 66, no. 4, pp. 2338-2345, 2006.

[25] L. A. Hazlehurst, N. Valkov, L. Wisner et al., "Reduction in drug-induced DNA double-strand breaks associated with $\beta 1$ integrin-mediated adhesion correlates with drug resistance in U937 cells," Blood, vol. 98, no. 6, pp. 1897-1903, 2001.

[26] F. Aoudjit and K. Vuori, "Integrin signaling inhibits paclitaxelinduced apoptosis in breast cancer cells," Oncogene, vol. 20, no. 36, pp. 4995-5004, 2001.

[27] J. H. Uhm, N. P. Dooley, A. P. Kyritsis, J. S. Rao, and C. L. Gladson, "Vitronectin, a glioma-derived extracellular matrix protein, protects tumor cells from apoptotic death," Clinical Cancer Research, vol. 5, no. 6, pp. 1587-1594, 1999.

[28] A. A. Ahmed, A. D. Mills, A. E. K. Ibrahim et al., "The extracellular matrix protein TGFBI induces microtubule stabilization and sensitizes ovarian cancers to paclitaxel," Cancer Cell, vol. 12, no. 6, pp. 514-527, 2007.

[29] E. Hutchinson, "Cancer stem cells: developmental block," Nature Reviews Cancer, vol. 8, no. 3, pp. 160-161, 2008.

[30] F. de Fraipont, A. C. Nicholson, J. J. Feige, and E. G. van Meir, "Thrombospondins and tumor angiogenesis," Trends in Molecular Medicine, vol. 7, no. 9, pp. 401-407, 2001.

[31] C. J. Lih, W. Wei, and S. N. Cohen, "Txr1: a transcriptional regulator of thrombospondin-1 that modulates cellular sensitivity to taxanes," Genes and Development, vol. 20, no. 15, pp. 2082-2095, 2006.
[32] R. van Amerongen and A. Berns, "TXR1-mediated thrombospondin repression: a novel mechanism of resistance to taxanes?" Genes and Development, vol. 20, no. 15, pp. 19751981, 2006.

[33] C. Papadaki, D. Mavroudis, M. Trypaki et al., "Tumoral expression of TXR1 and TSP1 predicts overall survival of patients with lung adenocarcinoma treated with first-line docetaxel-gemcitabine regimen," Clinical Cancer Research, vol. 15, no. 11, pp. 3827-3833, 2009.

[34] X. Peng, W. Li, and G. Tan, "Reversal of taxol resistance by cisplatin in nasopharyngeal carcinoma by upregulating thromspondin-1 expression," Anti-Cancer Drugs, vol. 21, no. 4, pp. 381-388, 2010.

[35] H. Y. Zhao, A. Ooyama, M. Yamamoto et al., "Molecular basis for the induction of an angiogenesis inhibitor, thrombospondin-1, by 5-fluorouracil," Cancer Research, vol. 68, no. 17, pp. 7035-7041, 2008.

[36] S. M. Hyder, Y. Liang, and J. Wu, "Estrogen regulation of thrombospondin-1 in human breast cancer cells," International Journal of Cancer, vol. 125, no. 5, pp. 1045-1053, 2009.

[37] S. M. Hyder, Y. Liang, J. Wu, and V. Welbern, "Regulation of thrombospondin-1 by natural and synthetic progestins in human breast cancer cells," Endocrine-Related Cancer, vol. 16, no. 3, pp. 809-817, 2009.

[38] G. Rath, C. Schneider, B. Langlois et al., "De novo ceramide synthesis is responsible for the anti-tumor properties of camptothecin and doxorubicin in follicular thyroid carcinoma," International Journal of Biochemistry and Cell Biology, vol. 41, no. 5, pp. 1165-1172, 2009.

[39] G. M. Rath, C. Schneider, S. Dedieu et al., "The C-terminal CD47/IAP-binding domain of thrombospondin-1 prevents camptothecin- and doxorubicin-induced apoptosis in human thyroid carcinoma cells," Biochimica et Biophysica Acta, vol. 1763, no. 10, pp. 1125-1134, 2006.

[40] H. El btaouri, H. Morjani, Y. Greffe, E. Charpentier, and L. Martiny, "Role of JNK/ATF-2 pathway in inhibition of thrombospondin-1 (TSP-1) expression and apoptosis mediated by doxorubicin and camptothecin in FTC-133 cells," Biochimica et Biophysica Acta, vol. 1813, no. 5, pp. 695-703, 2011.

[41] J. W. Lawler, H. S. Slayter, and J. E. Coligan, "Isolation and characterization of a high molecular weight glycoprotein from human blood platelets," Journal of Biological Chemistry, vol. 253, no. 23, pp. 8609-8616, 1978.

[42] N. L. Baenziger, G. N. Brodie, and P. W. Majerus, "A thrombinsensitive protein of human platelet membranes," Proceedings of the National Academy of Sciences of the United States of America, vol. 68, no. 1, pp. 240-243, 1971.

[43] B. Vailhé, D. Vittet, and J. J. Feige, "In vitro models of vasculogenesis and angiogenesis," Laboratory Investigation, vol. 81, no. 4, pp. 439-452, 2001.

[44] D. D. Roberts, "Regulation of tumor growth and metastasis by thrombospondin-1," FASEB Journal, vol. 10, no. 10, pp. 11831191, 1996.

[45] J. Savill, N. Hogg, Y. Ren, and C. Haslett, "Thrombospondin cooperates with CD36 and the vitronectin receptor in macrophage recognition of neutrophils undergoing apoptosis," Journal of Clinical Investigation, vol. 90, no. 4, pp. 15131522, 1992.

[46] J. M. Sipes, H. C. Krutzsch, J. Lawler, and D. D. Roberts, "Cooperation between thrombospondin-1 type 1 repeat peptides and $\alpha_{\mathrm{v}} \beta_{3}$ integrin ligands to promote melanoma cell spreading and focal adhesion kinase phosphorylation," Journal 
of Biological Chemistry, vol. 274, no. 32, pp. 22755-22762, 1999.

[47] T. N. Wight, G. J. Raugi, S. M. Mumby, and P. Bornstein, "Light microscopic immunolocation of thrombospondin in human tissues," Journal of Histochemistry and Cytochemistry, vol. 33, no. 4, pp. 295-302, 1985.

[48] H. Naganuma, E. Satoh, T. Asahara et al., "Quantification of thrombospondin-1 secretion and expression of $\alpha_{\mathrm{v}} \beta_{3}$ and $\alpha_{3} \beta_{1}$ integrins and syndecan-1 as cell-surface receptors for thrombospondin-1 in malignant glioma cells," Journal of Neuro-Oncology, vol. 70, no. 3, pp. 309-317, 2004.

[49] H. Chen, J. Sottile, D. K. Strickland, and D. F. Mosher, "Binding and degradation of thrombospondin-1 mediated through heparan sulphate proteoglycans and low-densitylipoprotein receptor-related protein: localization of the functional activity to the trimeric $\mathrm{N}$-terminal heparin-binding region of thrombospondin-1," Biochemical Journal, vol. 318, no. 3, pp. 959-963, 1996.

[50] M. J. Calzada, J. M. Sipes, H. C. Krutzsch et al., "Recognition of the N-terminal modules of thrombospondin-1 and thrombospondin-2 by $\alpha_{6} \beta_{1}$ integrin," Journal of Biological Chemistry, vol. 278, no. 42, pp. 40679-40687, 2003.

[51] J. Lawler and R. O. Hynes, "The structure of human thrombospondin, an adhesive glycoprotein with multiple calciumbinding sites and homologies with several different proteins," Journal of Cell Biology, vol. 103, no. 5, pp. 1635-1648, 1986.

[52] B. Jiménez, O. V. Volpert, S. E. Crawford, M. Febbraio, R. L. Silverstein, and N. Bouck, "Signals leading to apoptosis-dependent inhibition of neovascularization by thrombospondin-1," Nature Medicine, vol. 6, no. 1, pp. 41-48, 2000.

[53] M. D. Kosfeld and W. A. Frazier, "Identification of a new cell adhesion motif in two homologous peptides from the $\mathrm{COOH}$ terminal cell binding domain of human thrombospondin," Journal of Biological Chemistry, vol. 268, no. 12, pp. 88088814, 1993.

[54] C. Massart, R. Barbet, N. Genetet, and J. Gibassier, "Doxorubicin induces fas-mediated apoptosis in human thyroid carcinoma cells," Thyroid, vol. 14, no. 4, pp. 263-270, 2004.

[55] I. Sargiannidou, J. Zhou, and G. P. Tuszynski, "The role of thrombospondin-1 in tumor progression," Experimental Biology and Medicine, vol. 226, no. 8, pp. 726-733, 2001.

[56] H. U. Kasper, M. Ebert, P. Malfertheiner, A. Roessner, C. Kirkpatrick, and H. K. Wolf, "Expression of thrombospondin-1 in pancreatic carcinoma: correlation with microvessel density," Virchows Archiv, vol. 438, no. 2, pp. 116-120, 2001.

[57] D. S. Kim, J. A. Franklyn, A. L. Stratford et al., "Pituitary tumor-transforming gene regulates multiple downstream angiogenic genes in thyroid cancer," Journal of Clinical Endocrinology and Metabolism, vol. 91, no. 3, pp. 1119-1128, 2006.

[58] G. Bunone, P. Vigneri, L. Mariani et al., "Expression of angiogenesis stimulators and inhibitors in human thyroid tumors and correlation with clinical pathological features," American Journal of Pathology, vol. 155, no. 6, pp. 1967-1976, 1999.

[59] K. Tanaka, H. Sonoo, J. Kurebayashi et al., "Inhibition of infiltration and angiogenesis by thrombospondin-1 in papillary thyroid carcinoma," Clinical Cancer Research, vol. 8, no. 5, pp. 1125-1131, 2002.

[60] K. Maeda, Y. Nishiguchi, S. M. Kang et al., "Expression of thrombospondin-1 inversely correlated with tumor vascularity and hematogenous metastasis in colon cancer," Oncology Reports, vol. 8, no. 4, pp. 763-766, 2001.
[61] J. C. Rodríguez-Manzaneque, T. F. Lane, M. A. Ortega, R. O. Hynes, J. Lawler, and M. L. Iruela-Arispe, "Thrombospondin1 suppresses spontaneous tumor growth and inhibits activation of matrix metalloproteinase- 9 and mobilization of vascular endothelial growth factor," Proceedings of the National Academy of Sciences of the United States of America, vol. 98, no. 22, pp. 12485-12490, 2001.

[62] J. S. Isenberg, W. A. Frazier, and D. D. Roberts, "Thrombospondins: from structure to therapeutics Thrombospondin-1: a physiological regulator of nitric oxide signaling," Cellular and Molecular Life Sciences, vol. 65, no. 5, pp. 728-742, 2008.

[63] J. S. Isenberg, C. Yu, and D. D. Roberts, "Differential effects of ABT-510 and a CD36-binding peptide derived from the type 1 repeats of thrombospondin-1 on fatty acid uptake, nitric oxide signaling, and caspase activation in vascular cells," Biochemical Pharmacology, vol. 75, no. 4, pp. 875-882, 2008.

[64] L. S. Gutierrez, "The role of thrombospondin 1 on intestinal inflammation and carcinogenesis," Biomarker Insights, vol. 2008, no. 3, pp. 171-178, 2008.

[65] T. Nelius, S. Filleur, A. Yemelyanov et al., "Androgen receptor targets NF $\kappa$ B and TSP1 to suppress prostate tumor growth in vivo," International Journal of Cancer, vol. 121, no. 5, pp. 9991008, 2007.

[66] A. Gaurnier-Hausser, V. L. Rothman, S. Dimitrov, and G. P. Tuszynski, "The novel angiogenic inhibitor, angiocidin, induces differentiation of monocytes to macrophages," Cancer Research, vol. 68, no. 14, pp. 5905-5914, 2008.

[67] Y. A. Hannun, "The sphingomyelin cycle and the second messenger function of ceramide," Journal of Biological Chemistry, vol. 269, no. 5, pp. 3125-3128, 1994.

[68] C. Michel, G. van Echten-Deckert, J. Rother, K. Sandhoff, E. Wang, and A. H. Merrill Jr., "Characterization of ceramide synthesis. A dihydroceramide desaturase introduces the 4,5trans-double bond of sphingosine at the level of dihydroceramide," Journal of Biological Chemistry, vol. 272, no. 36, pp. 22432-22437, 1997.

[69] R. Kolesnick and Z. Fuks, "Radiation and ceramide-induced apoptosis," Oncogene, vol. 22, no. 37, pp. 5897-5906, 2003.

[70] M. Tani, M. Ito, and Y. Igarashi, "Ceramide/sphingosine/sphingosine 1-phosphate metabolism on the cell surface and in the extracellular space," Cellular Signalling, vol. 19, no. 2, pp. 229-237, 2007.

[71] V. V. Sumbayev and I. M. Yasinska, "Role of MAP kinasedependent apoptotic pathway in innate immune responses and viral infection," Scandinavian Journal of Immunology, vol. 63, no. 6, pp. 391-400, 2006.

[72] Y. Chovolou, W. Watjen, A. Kampkotter, and R. Kahl, "Downregulation of NF- $\kappa$ B activation in a H4IIE transfectant insensitive to doxorubicin-induced apoptosis," Toxicology, vol. 232, no. 1-2, pp. 89-98, 2007.

[73] B. W. Min, C. G. Kim, J. Ko, Y. Lim, Y. H. Lee, and S. Y. Shin, "Transcription of the protein kinase $\mathrm{C}-\delta$ gene is activated by JNK through c-Jun and ATF2 in response to the anticancer agent doxorubicin," Experimental and Molecular Medicine, vol. 40, no. 6, pp. 699-708, 2008.

[74] T. Noutomi, M. Itoh, H. Toyota, E. Takada, and J. Mizuguchi, "Tumor necrosis factor-related apoptosis-inducing ligand induces apoptotic cell death through c-Jun $\mathrm{NH}_{2}$-terminal kinase activation in squamous cell carcinoma cells," Oncology Reports, vol. 22, no. 5, pp. 1169-1172, 2009.

[75] M. Verheij, G. A. Ruiter, S. F. Zerp et al., "The role of the stress-activated protein kinase (SAPK/JNK) signaling pathway 
in radiation-induced apoptosis," Radiotherapy and Oncology, vol. 47, no. 3, pp. 225-232, 1998.

[76] K. Sengupta, S. Banerjee, N. K. Saxena, and S. K. Banerjee, "Thombospondin-1 disrupts estrogen-induced endothelial cell proliferation and migration and its expression is suppressed by estradiol," Molecular Cancer Research, vol. 2, no. 3, pp. 150-158, 2004.

[77] J. Liu and A. Lin, "Role of JNK activation in apoptosis: a double-edged sword," Cell Research, vol. 15, no. 1, pp. 36-42, 2005.

[78] L. Chang and M. Karin, "Mammalian MAP kinase signalling cascades," Nature, vol. 410, no. 6824, pp. 37-40, 2001.

[79] D. K. Morrison and R. J. Davis, "Regulation of map kinase signaling modules by scaffold proteins in mammals," Annual Review of Cell and Developmental Biology, vol. 19, pp. 91-118, 2003.

[80] M. Vacotto, O. Coso, and S. F. de Plazas, "Programmed cell death and differential JNK, p38 and ERK response in a prenatal acute hypoxic hypoxia model," Neurochemistry International, vol. 52, no. 4-5, pp. 857-863, 2008.

[81] M. Soula-Rothhut, C. Coissard, H. Sartelet et al., "The tumor suppressor PTEN inhibits EGF-induced TSP-1 and TIMP-1 expression in FTC-133 thyroid carcinoma cells," Experimental Cell Research, vol. 304, no. 1, pp. 187-201, 2005.

[82] S. Filleur, O. V. Volpert, A. Degeorges et al., "In vivo mechanisms by which tumors producing thrombospondin 1 bypass its inhibitory effects," Genes and Development, vol. 15, no. 11, pp. 1373-1382, 2001.

[83] N. Maruotti, F. P. Cantatore, E. Crivellato, A. Vacca, and D. Ribatti, "Angiogenesis in rheumatoid arthritis," Histology and Histopathology, vol. 21, no. 4-6, pp. 557-566, 2006.

[84] B. Ren, K. O. Yee, J. Lawler, and R. Khosravi-Far, "Regulation of tumor angiogenesis by thrombospondin-1," Biochimica et Biophysica Acta, vol. 1765, no. 2, pp. 178-188, 2006.

[85] A. Fontana, S. Filleur, J. Guglielmi et al., "Human breast tumors override the antiangiogenic effect of stromal thrombospondin-1 in vivo," International Journal of Cancer, vol. 116, no. 5, pp. 686-691, 2005.

[86] M. Yu and I. F. Tannock, "Targeting tumor architecture to favor drug penetration: a new weapon to combat chemoresistance in pancreatic cancer?" Cancer Cell, vol. 21, no. 3, pp. 327-329, 2012. 


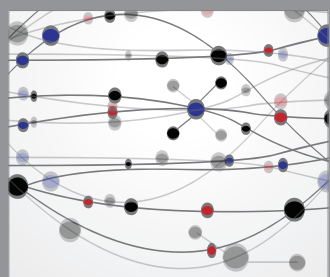

The Scientific World Journal
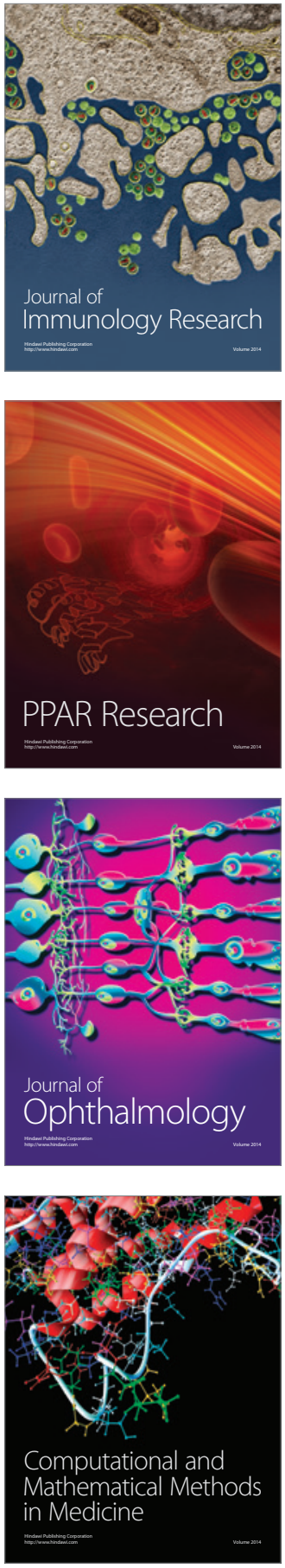

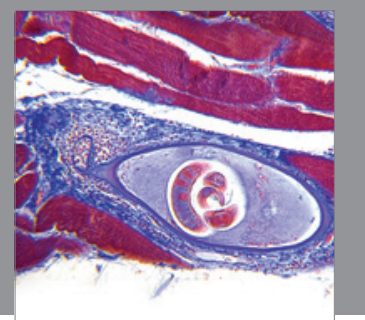

Gastroenterology

Research and Practice
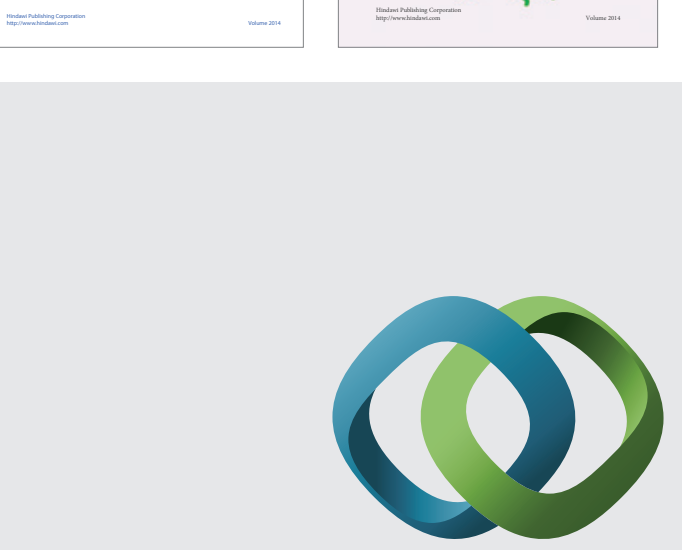

\section{Hindawi}

Submit your manuscripts at

http://www.hindawi.com
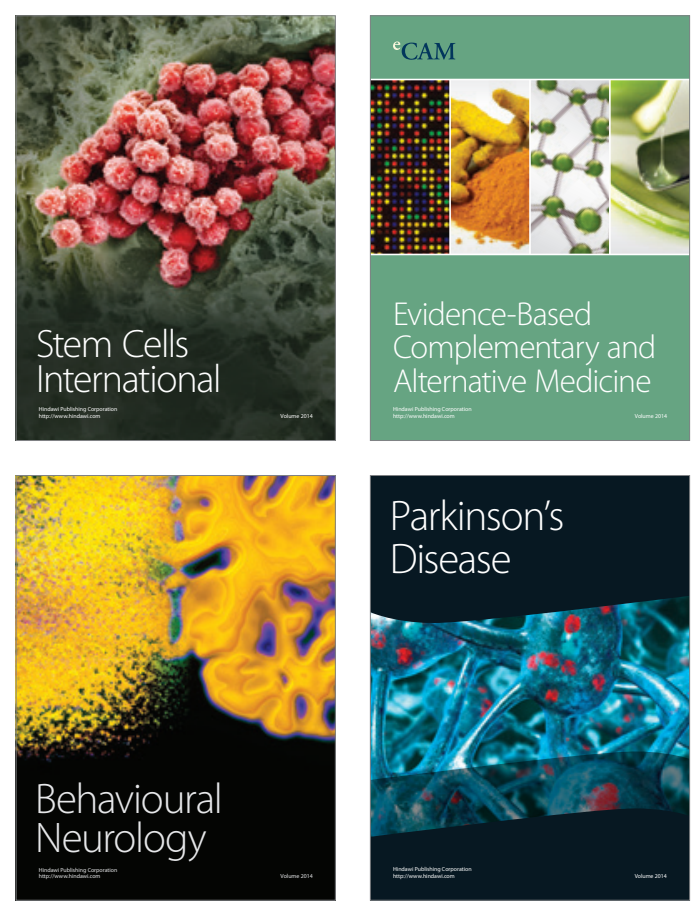

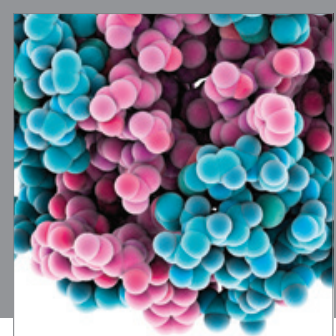

Journal of
Diabetes Research

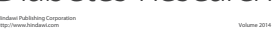

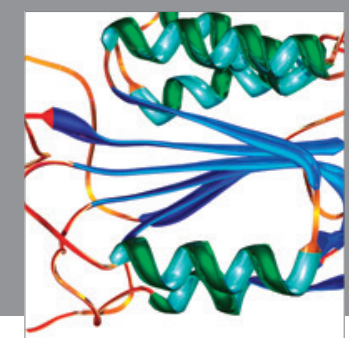

Disease Markers
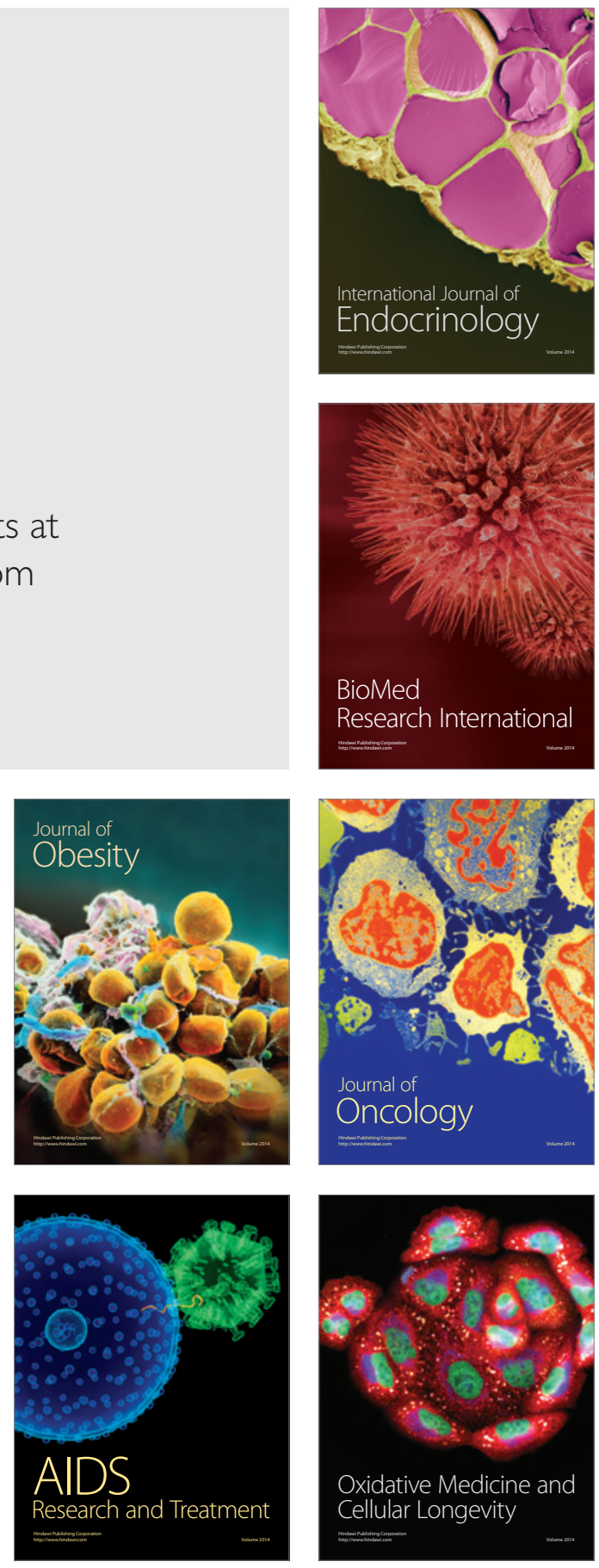